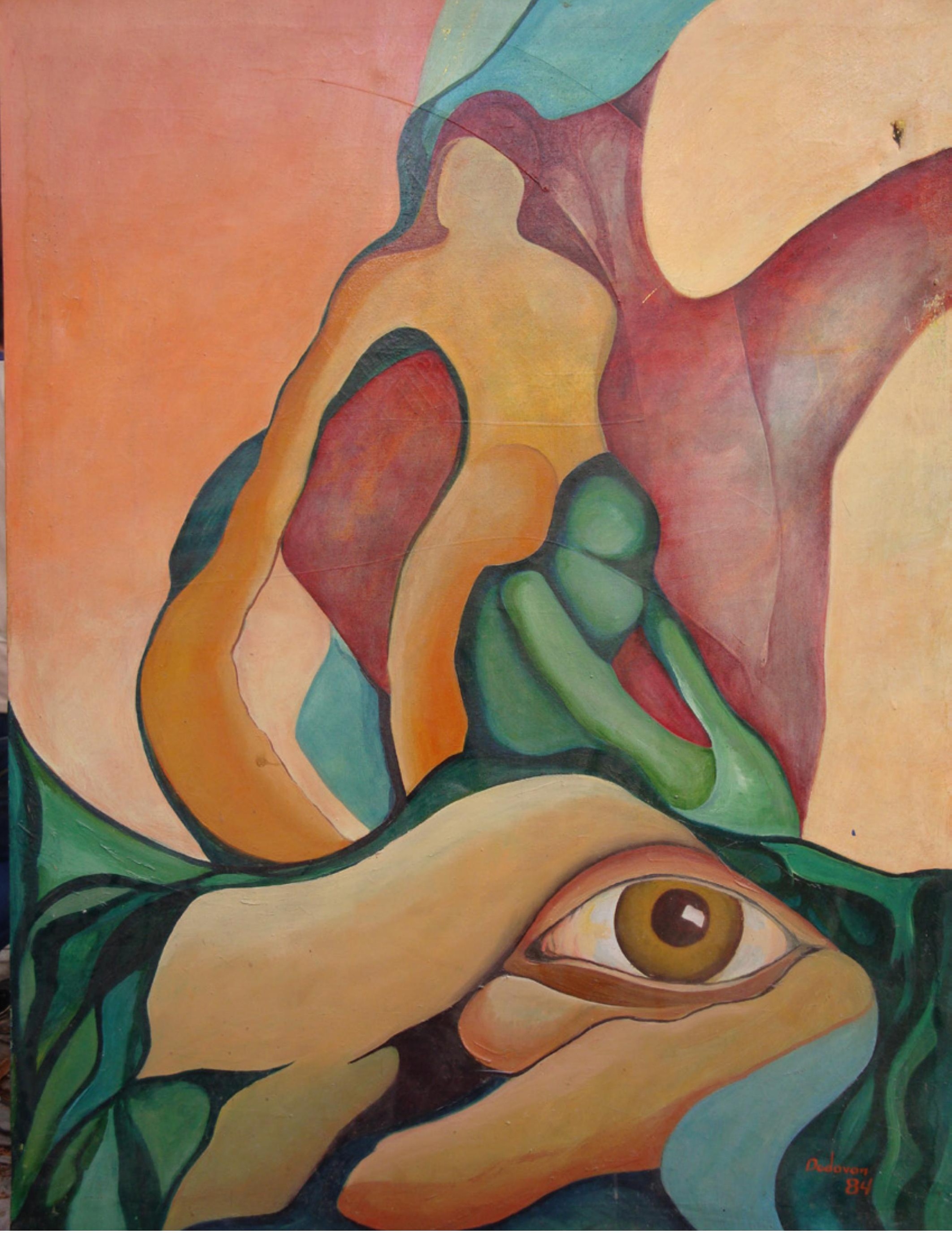




\section{Condiciones de las viviendas en la comunidad rural de Salado Barra, municipio de El Porvenir, Atlántida}

Kelly Johana Alemán Pineda

\section{INTRODUCIÓN}

"Investigar significa pagar la entrada por adelantado y

entrar

sin saber lo que se va a ver"

(Oppenheimer)

A mediados del mes de junio del año 2008, se comenzó un importante proceso de aprendizaje basado en la temática "Los Objetivos de Desarrollo del Milenio", propuestos en la Cumbre del Milenio realizada en la ciudad de Nueva York en el año 2000, dicho proceso se hizo através del Diplomado de en Objetivos de Desarrollo del Milenio con Énfasis en Análisis Sociodemográfico que logró enfatizar un conocimiento real y práctico de la situación que afronta y deberá lograr Honduras al año 2015 en aspectos sociales, educativos, culturales, ambientales y demográficos.

A partir de esta iniciativa realizada con el apoyo de la Universidad Nacional Autónoma de Honduras através de la Maestría en Demografía Social y como producto fundamental para culminar satisfactoriamente este proceso educativo se realizó un estudio que tiene como objetivo central describir en forma general las condiciones de la vivienda en relación a los materiales de construcción y saneamiento básico existente en la comunidad de Salado Barra, municipio de El Porvenir, Atlántida, mostrando estadísticamente las características de las mismas y su relación directa con el estado de salud de la población.

En el estudio se aborda de manera muy específica la particularidad de las viviendas, identificada a través de datos estadísticos obtenidos de fuentes de información secundaria, principalmente tomando como base el Censo de Población y Vivienda 2001; se realizaron algunas visitas que permitieron observar y recolectar información de manera directa.

\section{JUSTIFICACIÓN}

Cada día que transcurre, es posible visualizar que el problema de los asentamientos humanos y la vivienda en Honduras tiene muchos alcances, como en todos los países en desarrollo. En Honduras el déficit habitacional es enorme y afecta al 70\% de su población; lo que significa que más de tres millones y medio de personas residen en viviendas en condiciones precarias.

Según la Encuesta de Hogares de septiembre del 2007 realizada por el INE en Honduras, 2, 472,651 de las personas vive en extrema pobreza (42\%).Esta población pobre es la que no tiene acceso a la vivienda comercial y tampoco hay alternativas para mejorar su situación.

Estudios realizados por la Maestría en Demografía Social de Universidad Nacional Autónoma de Honduras, han reflejado que el $45 \%$ de las viviendas existentes en el país carecen de servicios de agua, energía eléctrica, y evacuación de excretas; y, el 54\% de las mismas correspondía a casas de un sólo dormitorio (MDS 2007).

En el caso especifico de Salado Barra, una comunidad eminentemente rural se identificó en el 2001, que el 93\% de las viviendas existentes corresponden a casas independientes, por lo general de un solo dormitorio; en tanto que el $97 \%$ de las mismas no tiene agua potable y ninguna posee acceso a alcantarillado sanitario, los 
niveles de hacinamiento son elevados, superan más del 50\% de las viviendas. (INE, 2001).

El desarrollo de este tipo de investigaciones pretende mostrar el problema de la vivienda en nuestro país, y específicamente en la comunidad de Salado Barra el objetivo es desarrollar un estudio que permita introducir expectativas orientadas a la priorización de alternativas a través de proyectos de mejoramiento de vivienda en aras de la reducción de la pobreza a nivel local, regional y de país.

\section{PLANTEAMIENTO DEL PROBLEMA}

Uno de los mayores problemas a nivel nacional y local es el déficit habitacional unido a las deficientes condiciones de las viviendas.

El presente estudio nos mostrará un análisis descriptivo de la situación real de las viviendas ubicadas en la comunidad de Salado Barra, municipio de El Porvenir. Este estudio servirá como referencia o punto de partida para el diseño de programas y proyectos orientados a mejorar la situación de los grupos más vulnerables, de la misma manera podrá ser utilizado por autoridades locales y organismos que tengan interés en la temática de estudio.

Corresponde a los gobiernos, tanto a nivel central como municipal establecer en sus planes de trabajo acciones que busquen aportar soluciones a los problemas que plantea la sociedad, y en especial a la problemática de vivienda.

El estudio en la comunidad de Salado Barra municipio de El Porvenir, Atlántida, permitirá conocer las condiciones en que vive la población respecto a sus características de sus viviendas; y servirá como referencia para ser evaluado por las autoridades municipales locales, organismos afines y de interés al tema objeto de estudio.

\section{OBJETIVOS}

\subsection{General}

Caracterizar la situación de la vivienda en la comunidad rural de Salado Barra, municipio de El Porvenir,
Atlántida en relación a los materiales de construcción y el acceso a saneamiento básico.

\subsection{Específicos}

1. Identificar los materiales de construcción utilizados en las viviendas de la comunidad de Salado Barra en el municipio de El Porvenir, según el Censo de Población y Vivienda del 2001.

2. Identificar el acceso a saneamiento básico que tienen las viviendas de la comunidad de Salado Barra en el municipio de El Porvenir, según datos del Censo de Población y Vivienda del 2001.

3. Establecer la relación que existe entre el estado de salud de la población y las características de las viviendas.

4. Identificar los programas y proyectos desarrollados para cubrir la necesidad de vivienda y/o mejoramiento de la misma, en la comunidad de Salado Barra.

\section{METODOLOGÍA UTILIZADA}

\subsection{Definición del tipo de estudio a realizar.}

El estudio es de tipo descriptivo, se desarrolló una revisión documental, en la cual se analizó toda la información escrita y recolectada sobre el tema de las condiciones de las viviendas en la comunidad de Salado Barra, con el fin de poder establecer relaciones, diferencias y sobre todo el estado actual en relación al tema de investigación planteado.

\subsection{Extracción de la muestra.}

Universo o población: Se tomó como unidades de estudio 40 viviendas habitadas por 309 personas, estas constituyen la totalidad de viviendas identificadas según datos obtenidos de fuentes documentales como ser el Censo de Población y Vivienda 2001 y censos realizados por organizaciones locales del municipio. El estudio se centró en una comunidad rural pequeña con poca población, lo que permitirá hacer estimaciones exactas a nivel local, reflejando datos confiables para la toma de decisiones futuras. 


\subsection{Revisión de la literatura}

Para el desarrollo de la investigación, el procedimiento o técnica usada en la recolección de información se realizó en base a la revisión y utilización de fuentes de información secundaria, utilizando bases de datos como ser las siguientes:

- Censo de Población y Vivienda 2001.

- Censo de Población realizado por Unidades de Salud del municipio.

- Diagnósticos socioeconómicos del municipio de El Porvenir.

- Diagnóstico del Sector Agua y Saneamiento.

- Plan Estratégico de Desarrollo Municipal 2003 -2010 .

- Plan Estratégico Municipal de Salud.

- Otros documentos que puedan aportar información relevante al tema.

VI. CONTEXTO GENERAL MUNICIPIO DE EL PORVENIR

\subsection{Ubicación}

El municipio de El Porvenir, se encuentra ubicado en la zona geofísica que conforman las tierras bajas costeras frente al Mar Caribe, denominado "Litoral Atlántico". Esta zona se caracteriza por ser estrecha, plana y fértil, y de suelos aluviales; además, es la zona con mayor precipitación pluvial de Honduras.

La posición geográfica del municipio de El Porvenir, Atlántida es latitud norte $15^{\circ} 45$ minutos y longitud oeste $86^{\circ} 53$ minutos.

El municipio se localiza sobre el litoral atlántico entre la desembocadura de los Ríos Juan Leandro y Coloradito, en la parte noreste a solo 12 Kilómetros sobre la carretera que conduce de La Ceiba hacia la ciudad capital.

La superficie territorial del municipio es de 280.26 $\mathrm{Km}^{2}$, equivalente a 27,250 hectáreas, de los cuales 3.75 $\mathrm{Km}^{2}$, corresponden al casco urbano y $276.51 \mathrm{Km}^{2}$ corresponden al área rural.

El municipio de El Porvenir cuenta con 13 barrios y colonias en el casco urbano, 12 aldeas y 53 caseríos en el área rural, en el municipio se hace necesario un ordenamiento territorial para determinar en forma exacta la cantidad de kilómetros cuadrados que corresponden al casco urbano y al área rural.

\section{Limites físicos del municipio}

Los límites geográficos del municipio de El Porvenir son:

- Al norte: con el Mar Caribe.

- Al sur: con el municipio de Olanchito, departamento de Yoro.

Figura No. 1. Mapa departamento de Atlántida por municipio

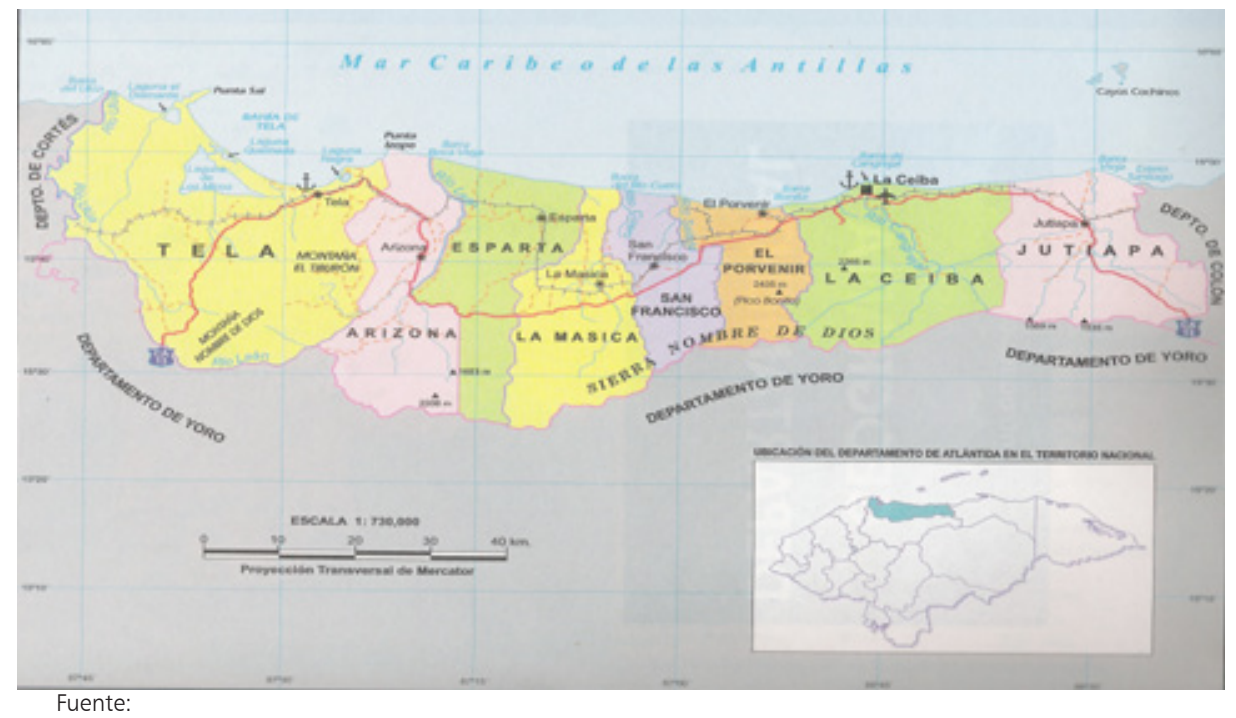


- Al este: con el municipio de La Ceiba.

- Al oeste: con el municipio de San Francisco.

El clima predominante en el municipio se clasifica como tropical húmedo, propio de la costa. Tiene aproximadamente $90 \mathrm{~km}^{2}$ de tierra plana y costera, y $180 \mathrm{~km}^{2}$ montañosa en la Sierra de Nombre de Dios, donde destaca una altura de 2,435 msnm, la más alta de la montaña Pico Bonito y la más alta frente al Mar Caribe, desde la Florida EE.UU. hasta Colombia.

\subsection{Datos poblacionales}

Según datos de la Unidad de Salud de El Porvenir en el 2008, el municipio cuenta con una población de 20,609 habitantes; el 60\% esta asentada en el área urbana que la constituyen El Porvenir (cabecera municipal), El Pino y La Unión; el resto de habitantes esta distribuido en las aldeas y caseríos. (US, 2008).

En el municipio de El Porvenir, todavía persisten los efectos negativos del huracán MITCH, por los daños que provocó a las principales represas abastecedoras de agua (El Porvenir, El Pino, La Unión y Monte Video) y todo

Figura No. 2. Mapa del municipio de El Porvenir por aldeas

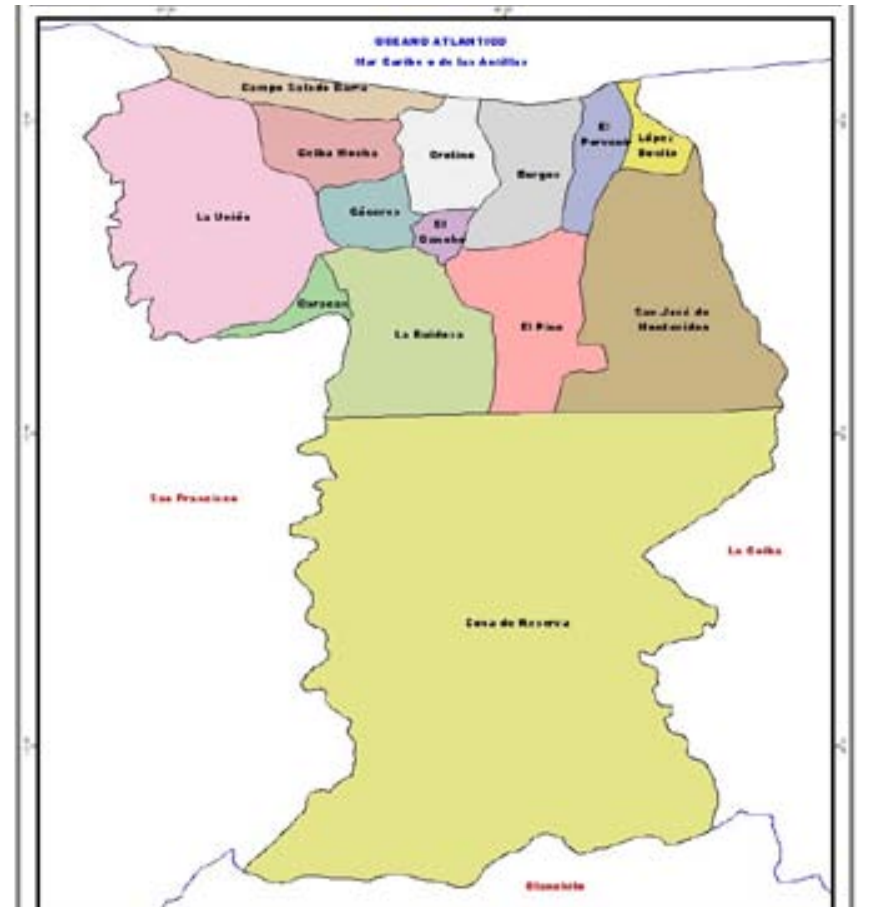

el sistema de agua potable; por otro lado, hubo daños a las vivienda y servicios básicos, el desempleo alcanzó un 50\% de la PEA condenando a vivir sin ingresos a la mitad de las familias del municipio.

\subsection{Patrimonio natural e histórico}

En el marco territorial del municipio de El Porvenir, se encuentra una reserva de vida silvestre, localizada a 17 kilómetros de la cabecera municipal en la costa del caribe, con una área en forma de triángulo constituida por los ríos de Cuero y Salado, esta área tiene una extensión de 13, 255 hectáreas, la cual fue declarada zona protegida en 1986, por estar en peligro de extinción, su población de manatíes, además tiene un complejo sistema de agua salobres y agua fresca y contiene unos 15 ríos que nacen en la montaña de Gracias a Díos, y que tienen influencia directa en el refugio en particular en el Parque de Pico Bonito.

De igual forma en El Porvenir, existe una verdadera joya natural que forma parte del patrimonio del municipio por ser el parque de Honduras más diverso cubriendo un área de terreno que va desde 7,300 pies sobre el nivel del mar con la mayor variación de altura de Honduras, ha sido nombrado Pico Bonito por su disposición y se ha convertido en un signo de la aldea El Pino, municipio de El Porvenir por el importante papel que juega con el ecoturismo. El principal sendero de esta área es el del río Zacate.

Figura No. 3. Mapa Refugio de vida silvestre Cuero y Salado

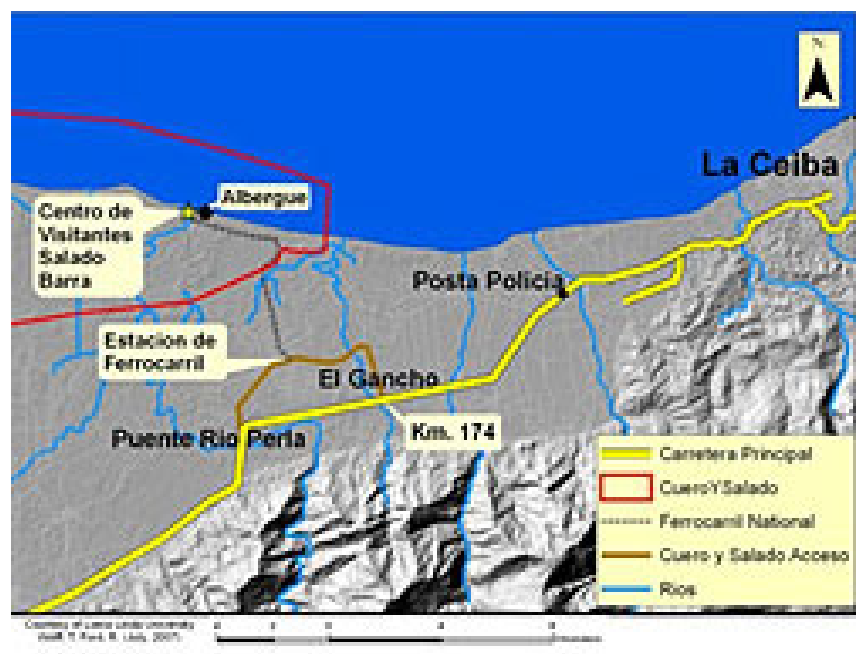




\subsubsection{Refugio de vida silvestre Cuero y Salado}

A 27 kilómetros de la ciudad de La Ceiba, entre los ríos Cuero y Salado, se localiza el Refugio de Vida Silvestre Cuero y Salado, con un área de 132.25 hectáreas que comprende 2 ecosistemas muy ricos en biodiversidad como lo son el bosque húmedo tropical y el bosque de manglar. Fue designado para la protección de especies amenazadas como el manatí y proteger los humedales.

Muchas de estas áreas de humedales y mangle de la costa norte de Honduras, han sido convertidos en pastos ganaderos o plantaciones agroindustriales, transformando la región debido a la deforestación.

Llegar al Refugio Cuero y Salado es fácil, ya que el acceso al mismo se encuentra viajando por automóvil y desliándose a la altura del Km 181 sobre la carretera de La Ceiba a Tela, se llega a la aldea de la Unión.

Aquí se aborda el histórico motocarro o "burra de línea", que transporta a los visitantes hacia el centro de información del área protegida.

El origen de su nombre se deriva de los ríos Cuero y Río Salado, los cuales atraviesan los ecosistemas de manglar y tierras bajas inundadas. Ubicado en el triángulo formado por la desembocadura de los ríos Cuero y Salado, está compuesto por una multitud de canales terrestres y fluviales. Recibe unos 15 ríos más y da albergue a gran cantidad de especies animales, algunas de ellas en peligro de extinción.

El Refugio de Vida Silvestre Cuero y Salado es el Primer Sitio Humedales RAMSAR de Honduras de importancia internacional, adscrito a esta lista de la ONU, designado el 23 de junio de 1993, bajo el No. 619. Quince de los ríos que se originan en la montaña de Nombre de Dios alimentan el refugio.

La Fundación Cuero y Salado (FUCSA), es la entidad encargada del manejo y protección del refugio. Se considera prioridad apoyar acciones encaminadas a mejorar las condiciones de vida de los habitantes y así reducir los efectos que estos tienen sobre los recursos del refugio. Este programa de desarrollo comunitario está estrechamente vinculado al programa de manejo integral de los recursos naturales.

\subsection{La comunidad de Salado Barra}

Dentro de las trece aldeas antes mencionadas, se encuentra la comunidad rural de Salado Barra, cuenta con una población de309 habitantes, según datos obtenidos en el censo realizado por unidades de salud municipal en el año 2008. Es una pequeña comunidad que se encuentra ubicada en la zona que comprende el Refugio de Vida Silvestre Cuero y Salado, área protegida contemplada dentro del municipio de El Porvenir, Atlántida, desde acá salen los senderos y las lanchas.

Los pobladores viven en medio de canales acuáticos, bosques de mangle, especies de aves y mamíferos en estado silvestre. Tiene muy fácil acceso a la playa y barras de los ríos, excepcionales para observación de aves al amanecer o al atardecer.

\subsubsection{Actividades económicas}

Salado Barra fue una zona habitada por los primeros Garifunas que llegaron a Honduras, después los olanchanos llegaron a trabajar con las compañías bananeras.

La principal actividad económica identificada en la comunidad de Salado Barra es la pesca artesanal, un pequeño grupo se dedica a la agricultura y la ganadería. En la comunidad habitan alrededor de 40 familias.

En relación a la actividad del turismo, los senderos acuáticos del refugio constituyen un importante potencial, que ofrece a los visitantes impresionantes vistas de hábitat y vida silvestre. Para un paseo con más calma por los canales acuáticos se ofrecen paseos en canoa con guías locales.

El visitante podrá escuchar con mayor precisión los sonidos de la naturaleza y observar la vida silvestre con mayor detenimiento.

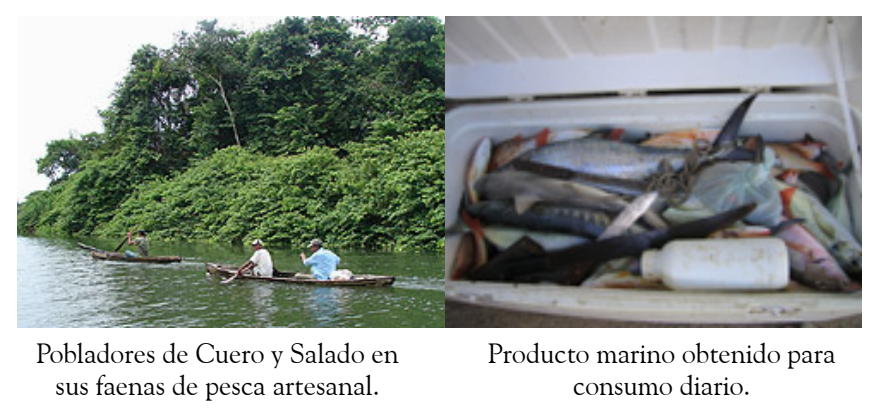




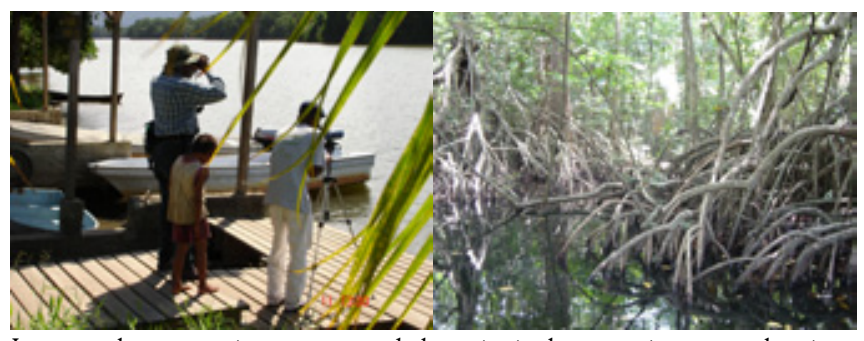

Los mangles se convierten en uno de los principales atractivos para el turista.

Los pobladores del refugio, especialmente aquellos quienes han sido capacitados y preparados como guías locales desarrollan esta importante actividad con los turistas, y a quienes llevan en sus tradicionales medio de transporte "cayucos" a través de los canales acuáticos para pescar un buen róbalo con la opción de prepararlo a la hora del almuerzo.

El tour en lancha es una de las más solicitadas por los visitantes para recorrer los distintos senderos acuáticos.

Esta actividad se convierte en otra significativa fuente de ingreso para algunos de los pobladores, sin embargo, no todos tienen acceso a formar parte del equipo de guías locales, dada su nivel de formación y disponibilidad de un transporte seguro que ofrecer; por lo general, esta actividad es manejada a través de la Fundación Cuero y Salado, quienes cuentan con la logística necesaria para desarrollarla.

\subsection{Antecedentes históricos}

Entre 1860 y 1870 Salado Barra se convirtió en uno de los más fuertes y comerciales embarcaderos de las compañías bananeras.

En 1908, llegó la primera locomotora de vapor a La Ceiba procedente de Salado Barra a través de la línea férrea que la Vaccaro Brothers construyó. La comunidad tiene un potencial turístico dado por la condición de estar

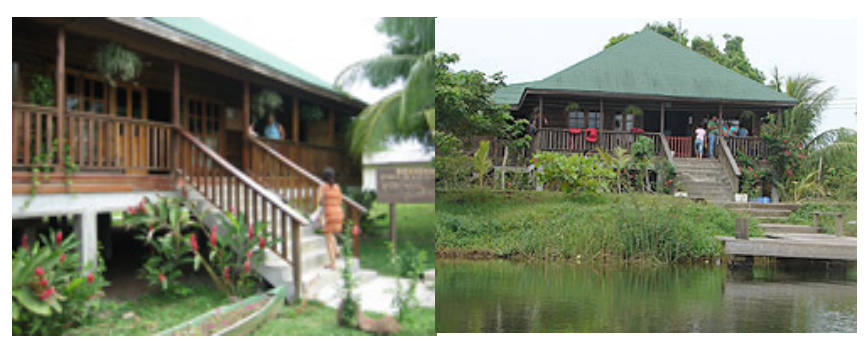

Edificio del Centro para los Visitantes en la comunidad de Salado Barra. en la zona del primer humedal de Honduras, cuya finalidad es la conservación de la flora y fauna que en el habita; no así, la participación comunitaria en las actividades propias de desarrollo del turismo es relativamente poca.

Actualmente, la comunidad de Salado Barra sólo cuenta con un centro de salud, con escaso personal y material para atención de la población; habilitado una vez por semana y con personal de enfermería proveniente de aldeas cercanas como La Unión.

Cuenta con un centro de visitantes, conformado con una sala de interpretación, tienda de souvenir, cafetería, sala de eventos donde se brinda información turística del refugio.

Dadas estas y otras condiciones prevalecientes, se hace necesario enfocar un estudio ante la necesidad de dar a conocer la situación actual y condición de la vivienda en esta comunidad, sus efectos en la salud de la población y las alternativas posibles para mejorar la calidad de vida de la misma.

VII. CONDICIONES DE LAS VIVIENDAS EN LA COMUNIDAD RURAL DE SALADO BARRA, MUNICIPIO DE EL PORVENIR, ATLANTIDA.

La vivienda es el local de alojamiento, estructuralmente separado e independiente, dedicado para la habitación humana.

En la comunidad de Salado Barra, las viviendas tienen características en común en relación a tamaño y materiales predominantes utilizados para su construcción, panorama inicial que suele apreciarse al ingresar a la misma, esto la convierte en una típica y tradicional comunidad. Dicho panorama refleja una comunidad que al igual que otras, se encuentra en situación de pobreza.

Las actividades que representan un ingreso económico para los habitantes de la misma, son precarias y están orientadas principalmente a la economía informal y de subsistencia, lo que significa que los ingresos por tales actividades son bajos.

Es evidente que este y otros factores contribuyen considerablemente a que los hogares de esta comunidad no 


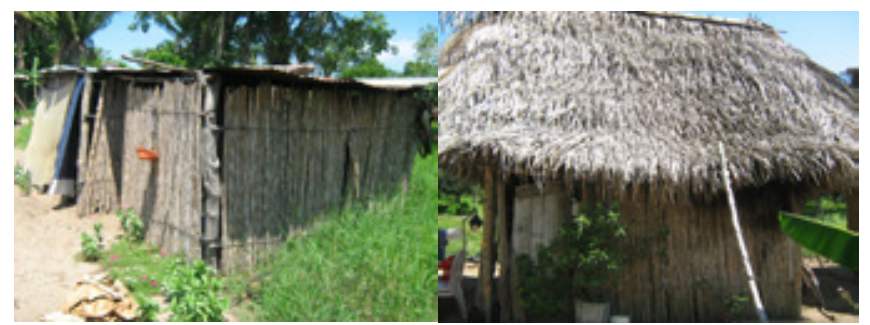

Viviendas con techo de paja y láminas de zinc, en la comunidad de Salado Barra.

puedan satisfacer algunas de las necesidades básicas, como ser: acceso a una vivienda digna y saneamiento básico.

\subsection{Materiales de construcción de la vivienda}

- Techo de las viviendas.

En Honduras, la caracterización del contexto rural esta determinado por las condiciones de vulnerabilidad demográfica y social.

En relación a las condiciones de la viviendas en el área rural los materiales utilizados en la construcción de techos son: láminas de zinc, asbesto o de fibrocemento, tejas de barro, material de desecho, paja o palma y plancha de cemento (Población y Desarrollo, 2003).

En el desarrollo de este trabajo se ha logrado identificar que la mayoría de las casas en la comunidad de Salado Barra son autoconstruidas y se caracterizan por tener techos de palma y en otros casos los techos de zinc se encuentran en mal estado; en general la construcción tradicional depende básicamente de las habilidades de los habitantes y la disposición de materiales autóctonos.

Esto a su vez, resulta directamente de la condición social, económica y educacional de la población que en ella habita.

Según información censal del 2001, en la comunidad de Salado Barra el 41.94\% de 31 viviendas registradas utilizaban techo de palma o paja, cifra que supera el dato a nivel del municipio en gran magnitud, en tanto que el $54.84 \%$ hacen uso de láminas de zinc; ambas cifras presentan una diferencia de $12.90 \%$ (Cuadro No. 1).

La significativa prevalencia de techos a base de láminas de zinc y paja en esta comunidad, se atribuye a los

$\begin{aligned} & \text { Cuadro No. 1. Materiales de construcción utilizados } \\
& \text { en los techos de las viviendas de la comunidad de } \\
& \text { Salado Barra }\end{aligned}$
\begin{tabular}{|l|c|c|}
\hline Categorías & Total & $\%$ \\
\hline Lamina de asbesto & 1 & 3.22 \\
\hline Lamina de zinc & 17 & 54.84 \\
\hline Paja, palma o similar & 13 & 41.94 \\
\hline TOTAL & 31 & 100 \\
\hline
\end{tabular}

Fuente: Elaboración propia en base a los datos del Censo 2001.

bajos costos en relación a otros materiales que pudiesen utilizar para el mismo fin, sin embargo, ambos presentan algunas desventajas en relación a las condiciones de bienestar de las familias, en el caso de los techos de paja, dado que en verano y en épocas lluviosas este material es susceptible y frágil a la humedad por lo que no brinda la protección deseada, pudiéndose a la vez convertir en un posible receptor de insectos y zancudos que en su defecto dañan la salud de los pobladores.

De igual manera, los techos con láminas de zinc suelen ser vulnerables a estados climáticos lluviosos con vientos si este no se sujeta bien a la estructura de la vivienda.

Es notable que en esta comunidad se realiza una lucha por mejorar estas condiciones, indicador de ello se ve reflejado en la utilización de materiales más seguros como el zinc, sin embargo, puede aludirse que existen viviendas que utilizan para sus techos materiales deplorables y frágiles.

\section{- Paredes de las viviendas}

En Honduras, los materiales más utilizados en la construcción de las paredes de la vivienda son: el bloque, adobe, ladrillo rafón, madera, material de desecho y el bahareque.

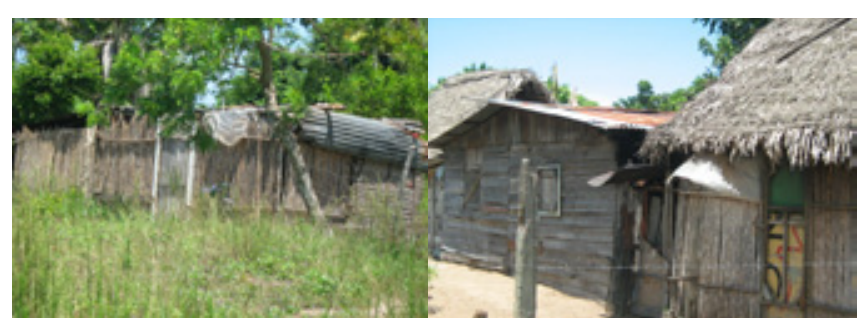

Viviendas construidas con diversos tipos de material en las paredes. 
Los datos censales del 2001 muestran que en la comunidad de Salado Barra, las paredes de las viviendas son diversas en relación a los materiales utilizados; existen casas con paredes de bloque de cemento, otras de palo o caña, como material predominante en la mayoría de los casos construidas a base de madera.

Sin embargo, existen viviendas que son autoconstruidas con una variedad de materiales de desecho para las paredes, entre ellos cartón, plásticos, etc. (Cuadro No. 2)

Dada la situación de pobreza en que viven estos pobladores de la comunidad de Salado Barra, se presentan grandes dificultades para mejorar las condiciones de la vivienda en relación a la paredes, por lo que estos deben hacer uso de su fuerza de trabajo para obtener material local, tratando de buscar una segura pero vulnerable condición que les permita proteger a su familia, lo que representa el fruto de su esfuerzo diario por mejorar sus condiciones de vida.

Esta condición a su vez les resulta factible por el bajo costo, pero se convierte en un riesgo en situaciones vulnerables al ambiente y en condiciones de salud representa un incipiente y fácil alojamiento de diversos tipos de animales que ponen en peligro la vida de la familia y en especial de los niños y niñas.

- Piso de las viviendas

Según el Instituto Nacional de Estadística (2001), los materiales más comunes empleados en los pisos de las viviendas en Honduras son los siguientes: plancha de cemento, madera, ladrillo de barro, ladrillo de granito y la cerámica.

Cuadro No. 2. Materiales de construcción utilizados en las paredes de las viviendas de la comunidad de Salado Barra

\begin{tabular}{|l|c|}
\hline Categorías & Total Casos \\
\hline Bloque de cemento & 5 \\
\hline Madera & 14 \\
\hline Palo o caña & 12 \\
\hline TOTAL & 31 \\
\hline Fuente: Elaboración propia en base a los datos del Censo 2001
\end{tabular}

El piso como tal cumple una importante función al interior y exterior de la vivienda, es simplemente la superficie de una habitación y puede estar en cualquier nivel del edificio (a nivel del suelo, primer piso, etc).

El piso que predomina en las viviendas de la comunidad de Salado Barra es el de tierra, según estadísticas del censo 2001, el 60\% de las mismas poseen este tipo de piso; en segundo lugar un $23.33 \%$ de las viviendas tienen piso de madera y sólo un $16.67 \%$ utilizan plancha de cemento en los pisos. (Cuadro No. 3)

Esta característica esta determinada en su defecto y como antes se ha mencionado, por la situación social y económica de los pobladores, la escasa disponibilidad de recursos limitan a estos pobladores a emprender actividades orientadas a mejorar esta situación, sumado a ello, el alto costo de materiales como el cemento y arena para aspirar a tener un piso más saludable y en donde se pueda desarrollar una vida digna.

\section{Gráfico No. 1. Materiales de construcción utilizados en las paredes de las viviendas de la comunidad} de Salado Barra

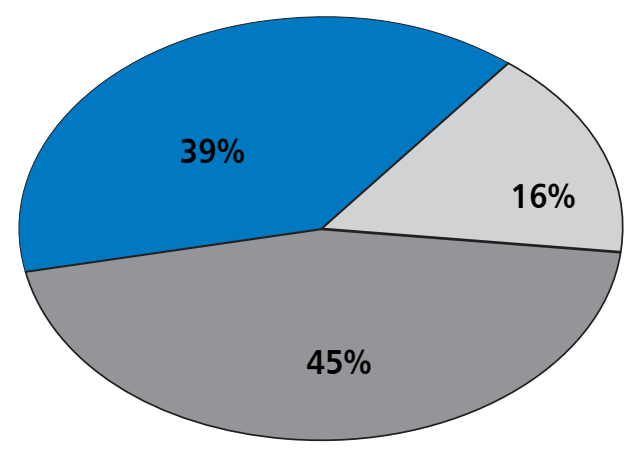

Bloque de cemento $\square$ Madera

Palo o caña

Fuente: Elaboración propia en base a los datos del Censo 2001.

\begin{tabular}{|c|c|c|}
\hline Categorías & Total & $\%$ \\
\hline Tierra & 18 & 60.0 \\
\hline Plancha de cemento & 5 & 16.6 \\
\hline Madera & 7 & 23.3 \\
\hline TOTAL & 30 & 100 \\
\hline
\end{tabular}

Fuente: Elaboración propia en base a los datos del Censo 2001 


\subsection{Saneamiento básico en la comunidad de Sa-} lado Barra

El agua potable es indispensable para la vida de los seres humanos, pero escasea en la medida que la población aumenta y porque lamentablemente es desperdiciada por personas carentes del sentido de responsabilidad y solidaridad humana.

El agua es un elemento indispensable para la existencia de los seres humanos por eso es preocupante que su obtención y conservación se esté convirtiendo en un problema crucial para las autoridades locales y nacionales.

En nuestro país, existen problemas de agua potable tanto en el área urbana como en el área rural generalmente estos se relacionan con problemas de abastecimiento y suministro y con la mala calidad de servicio.

Según información obtenida a través del Diagnóstico de Prestación de Servicios de Agua y Saneamiento en el municipio de El Porvenir, Atlántida, realizado en el año 2007, se demostró a que a nivel del municipio Salado Barra constituía la única comunidad en donde la cobertura del servicio es muy reducida, es decir la mayor parte de la comunidad no cuenta con servicio de acueductos y agua potable. (ANEXO 1), situación que les obliga a abastecerse de fuentes como pozo malacate o pozo con bomba hidráulica, lo que implica un acarreo diario del vital liquido.

La situación de pobreza, entre otros aspectos, es un factor que incide o dificulta la obtención de este beneficio, sin embargo, los pobladores de Salado Barra, han realizado numerosos esfuerzos que les permita contar con un sistema de agua potable para facilitar el desarrollo de sus labores diarias en el hogar.

En el año 2006, se logró un primer intento por mejorar esta situación, con el apoyo de organismos internacionales cooperantes como la Agencia Española de Cooperación Internacional - AECI quien inició la construcción de un tanque de agua potable para la comunidad. Sin embargo, hasta el momento no se ha logrado el resultado previsto.

Los datos del Censo de Población y Vivienda del 2001, demuestran que en la comunidad de Salado Barra el servicio de distribución de agua potable se realiza principalmente a través de pozo malacate representado por un $50 \%$ de las viviendas registradas, seguido a este, un $33 \%$ se abastece de tubería del sistema público/privado, en tanto que un $17 \%$ obtiene este beneficio a través de pozo con bomba.

En relación a la disponibilidad de tubería para el servicio de agua potable, se logró identificar que básicamente las viviendas tienen este sistema fuera de la misma y otra significativa proporción no cuenta con un sistema de tubería en sus hogares. Tan sólo un 13.33\% cuenta con este dentro de la vivienda. (Cuadro No. 4)

El agua que utilizan los pobladores depende básicamente de la disponibilidad del pozo malacate, dado el nivel freático a que se encuentra la comunidad, es destinada en su mayor parte a la realización de las labores domésticas y consumo humano, esto a su vez se convierte en un alto riesgo en la salud de los mismos, siendo que el agua que se utiliza para tal fin no es tratada, por lo que esta no reúne las condiciones de salubridad necesarias para el consumo, sin embargo, la imperante necesidad y la

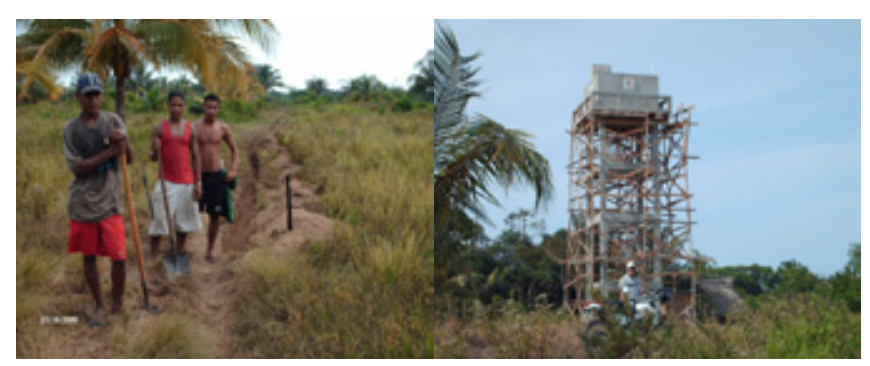

El aporte comunitario fue importante para la construcción del tanque de agua potable.

\begin{tabular}{|c|c|c|}
\hline Categorías & Total & $\%$ \\
\hline Dentro de la vivienda & 4 & 13.33 \\
\hline Fuera de la vivienda & 13 & 43.33 \\
\hline No tiene & 13 & 43.33 \\
\hline TOTAL & 30 & 100 \\
\hline
\end{tabular}

Fuente: Elaboración propia en base a los datos del Censo 2001 
irregularidad del servicio de agua obliga a los pobladores a seguir haciendo uso cotidiano de la misma.

\section{- Eliminación de excretas.}

La comunidad de Salado Barra no cuenta con un servicio de acueductos y alcantarillado, la eliminación de excretas en la mayoría de las casas de realiza a través del uso de letrinas y en su defecto al aire libre, situación que contribuye a la propagación de enfermedades gastrointestinales.

\section{- Eliminación de la basura}

El municipio de El Porvenir, Atlántida, a nivel general carece de un medio de recolección de la basura para las comunidades, no cuenta con un relleno sanitario que permita solventar la problemática.

Según datos registrados por el Censo de Población y Vivienda del 2001el 99.5\% de las viviendas de esta comunidad queman o entierran la basura; esta situación contribuye a la contaminación ambiental en continuo crecimiento, riesgo de incendios y conlleva a su vez a la proliferación de enfermedades en la comunidad.

- Estado de salud de la población de Salado

\section{Barra}

En la comunidad de Salado Barra funciona un centro de salud cofinanciado administrativamente por la Secretaria de Salud, ahora con el apoyo de la actual Corporación Municipal 2006-2010; el centro brinda los servicios de consulta general, a la vez se desarrollan diversos programas de salud entre ellos: vacunaciones, control de embarazo, planificación familiar, etc.

El centro cuenta únicamente con una enfermera auxiliar que reside en la zona, esta misma realiza una visita semanal a la comunidad para atender los casos pertinentes.

Datos recabados en visita realizada al centro de salud, dieron a conocer algunas estadísticas de la situación actual de la población en relación a la salud; se logró determinar que en el año 2008 se presentaron 154 atenciones nuevas registradas en el centro de salud, es decir un 50\% de la población ha acudido a este, dichas atenciones son básicamente coincidentes con el patrón de morbilidad.
En relación al control de mujeres embarazadas, en esta comunidad se tienen registrados 3 casos en el año 2008, representa menos del 1\% de la población, sin embargo, sólo 2 de estos casos reciben atención puerperal.

En el programa de planificación familiar, apenas 40 personas han sido registradas, que representa un 13\% de la población de la comunidad. (Registros administrativos, Centro de Salud, Salado Barra, 2008).

Es importante hacer notar que además de contar con el apoyo de una enfermera en la comunidad, comités locales de los otros centros de salud del municipio realizan visitas mensuales para desarrollar jornadas de vacunación.

El personal que labora en el centro de salud es de la opinión que la visita a la comunidad sólo se realiza una vez por semana dado que no se justifica el costo operativo, ya que las atenciones diarias en algunos casos es menor a 2 pacientes.

Por lo general, en la comunidad de Salado Barra, la mayor parte de las atenciones registradas en el centro de salud son por consulta general y el patrón de son por morbilidad esta directamente relacionado con enfermedades gastrointestinales que generalmente se manifiestan con diarreas.

En relación a la salud, como indicador de desarrollo, es evidente que las condiciones de la vivienda es un factor determinante en el estado de salud de la población, puesto que las enfermedades que padece la población están directamente relacionadas con la escasa disponibilidad de servicios básicos adecuados (baja calidad del agua), y de alguna manera la precariedad en las condiciones de las viviendas (paredes, pisos de tierra, techo).

Un detalle que vale la pena mencionar es que la población acostumbra a automedicarse dada la carencia de recursos económicos y las pocas opciones para adquirir medicamentos adecuados para tales casos.

\subsection{Programas y proyectos de vivienda}

En la comunidad de Salado Barra se ha identificado que es poco el apoyo que se brinda en relación al mejoramiento de la vivienda, hasta la fecha se desconoce la 
iniciativa por parte de organismos locales e internacionales que hayan realizado programas o proyectos orientados a este fin; sin embargo, los esfuerzos por contribuir a mejorar las condiciones habitacionales de esta población sigue siendo una lucha constante, logrando que poco se solventen parte de los problemas que enfrenta la comunidad.

En relación a la vivienda, aún no se concreta la iniciativa para mejorar una o varias características de la misma. La actual Corporación Municipal de El Porvenir 2006-2010, ha realizado gestiones ante organismos nacionales, a través fondos de la Estrategia para la Reducción de la Pobreza (ERP), destinados a reducir la vulnerabilidad habitacional de las familias de esta comunidad, proveyendo un techo digno y seguro, por lo que se espera que con el respaldo de dichos fondos estos pobladores puedan recibir material como ser láminas de zinc para mejorar sus viviendas.

\section{CONCLUSIONES}

- La imperante característica de la vivienda en la comunidad de Salado Barra, donde predominan techos de paja, paredes de madera, piso de tierra y escaso saneamiento básico es un indicador de la falta de oportunidades de los pobladores de la misma; dado por la prevalencia de ocupaciones del sector informal que no permite una estabilidad económica para hacer frente a la problemática generada en su diario vivir.

- En términos de cobertura del servicio de abastecimiento de agua potable y saneamiento básico, la comunidad de Salado Barra alcanza los niveles más bajos en el municipio de El Porvenir, Atlántida, lo que demuestra que las políticas locales de inversión en acueductos y agua potable no han sido priorizadas como tal, es necesario concentrar mayor atención y recursos económicos en esta comunidad a efecto de contribuir a alcanzar los Objetivos de Desarrollo del Milenio al 2015.

- Promover la integración de esta investigación a otros estudios en desarrollo o futuros programas de instituciones de investigación, universidades especialmente en lo relacionado con aspectos epidemiológicos y socioeconómicos en general.

- Es importante señalar la contribución del presente estudio al rescatar información base para orientar acciones en un proceso de construcción y desarrollo de esta comunidad, lo que contribuiría a disminuir el nivel de pobreza, logrando así mismo mayor capital humano a nivel familiar y comunal.

\section{BIBLIOGRAFÍA}

- Borjas, B. (2007) Diagnostico del Sector de Agua y Saneamiento, Municipio de El Porvenir, Atlántida.

- INE, (2001) Base del Censo de Población y Vivienda

- Plan Estratégico de Desarrollo Municipal, El Porvenir, Atlántida. 2006

- Plan Estratégico Municipal de Salud, El Porvenir, Atlántida. 2003

- Registros estadísticos, clínicos, Unidad de Salud de El Porvenir, Atlántida. 2008.

- Suazo, M., Ochoa, M., et al, Discusión critica de tres métodos de medición de la pobreza, Tegucigalpa. 2005.

- $\quad$ www.latribuna.hn/data/images/salado 